\title{
Integrability and Conservation Laws for the Nonlinear Evolution Equations of Partially Coherent Waves in Noninstantaneous Kerr Media
}

\author{
T. Hansson, M. Lisak, and D. Anderson \\ Department of Earth and Space Sciences, Chalmers University of Technology, SE 41296 Göteborg, Sweden
}

(Received 8 July 2011; published 8 February 2012)

\begin{abstract}
It is shown that the evolution equations describing partially coherent wave propagation in noninstantaneous Kerr media are integrable and have an infinite number of invariants. A recursion relation for generating these invariants is presented, and it is demonstrated how to express them in the coherent density, self-consistent multimode, mutual coherence, and Wigner formalisms.
\end{abstract}

DOI: 10.1103/PhysRevLett.108.063901

PACS numbers: 42.65.Tg, 42.65.Sf

A crucial step in the understanding and description of the properties of the nonlinear propagation of coherent waves was the development of the inverse scattering theory [1]. This made it possible to find general solutions for a broad class of nonlinear evolution equations for arbitrary initial conditions. Unique features of this solution procedure are the Zakharov-Shabat eigenvalue problem [2], the Lax formalism [3], and the fact that the integrability of the system is manifested by the existence of an infinite number of invariants, i.e., conserved quantities. In particular, the well-known soliton solution was first thought to be an inherently coherent phenomenon until it was recently found both experimentally [4] and theoretically [5] that similar solitonlike wave structures can also exist for partially coherent waves in noninstantaneous nonlinear media. Although at least four different but equivalent [6,7] approaches have been developed for describing the nonlinear propagation of partially coherent waves in terms of characteristic evolution equations [8], no general formalism, analogous to the inverse scattering theory, has been developed for the nonlinear evolution equations describing nonlinear propagation of partially coherent waves.

The purpose of the present Letter is to contribute to the development of the theory of nonlinear propagation of partially coherent waves in noninstantaneous Kerr media, by providing proper generalizations of the classical concepts of the Zakharov-Shabat eigenvalue problem and the Lax formalism, and to give an explicit recursion formula for the generation of an infinite number of invariants. It is also shown how these invariant quantities can be expressed in the coherent density [5], self-consistent multimode [9], mutual coherence, and Wigner formalisms [10,11]. These results should provide an important step forward in the investigation of the dynamics of partially coherent waves in nonlinear media, with the conservation laws finding multiple uses in the theoretical analysis of solutions, providing information on, e.g., evolution, stability, and soliton decomposition. Invariant quantities are further useful as a way of assessing the accuracy of many numerical schemes. In addition to various applications in the area of nonlinear optics $[8,12-14]$, the obtained results should also be of interest to a number of scientific disciplines such as biophysics [15], Bose-Einstein condensates [16], plasma physics [17], and water waves [18-20], where the coupled nonlinear Schrödinger system of the self-consistent multimode theory and the evolution equations of some of the other equivalent formalisms are used as model equations.

The propagation of partially coherent light in a noninstantaneous nonlinear Kerr medium can be described by the coherent density function $f(x, z, \theta)$ satisfying the evolution equation [5]

$$
i\left(\frac{\partial f}{\partial z}+\theta \frac{\partial f}{\partial x}\right)+\beta \frac{\partial^{2} f}{\partial x^{2}}+\kappa\left(\int_{-\infty}^{\infty}|f|^{2} d \theta\right) f=0,
$$

where $z$ is the distance of propagation, $x$ is the transverse field coordinate, $\theta$ represents an angle with respect to the propagation axis, and $\beta$ and $\kappa$ are diffraction and nonlinear coefficients, respectively. Using the transformation $f(x, z, \theta) \exp \left(i \frac{\theta}{2 \beta} x-i \frac{\theta^{2}}{4 \beta} z\right) \rightarrow|\psi(x, z ; \theta)\rangle$, where $|\psi\rangle$ is interpreted as an infinite-dimensional column vector labeled by the continuous index $\theta$, Eq. (1) can conveniently be written using a bra-ket notation as

$$
i \frac{\partial}{\partial z}|\psi\rangle+\beta \frac{\partial^{2}}{\partial x^{2}}|\psi\rangle+\kappa\langle\psi \mid \psi\rangle|\psi\rangle=0,
$$

with $\langle\psi|$ being the Hermitian conjugate row vector, and the integral is replaced by the scalar product $\left\langle\psi_{1} \mid \psi_{2}\right\rangle=$ $\int_{-\infty}^{\infty} \psi_{1}^{*} \psi_{2} d \theta$. The identification of Eq. (1) with the vector problem Eq. (2) is key to the subsequent analysis.

For a finite-dimensional ket vector $|\psi\rangle$, Eq. (2) reduces to a system of coupled nonlinear Schrödinger equations, viz.,

$i \frac{\partial \psi_{n}}{\partial z}+\beta \frac{\partial^{2} \psi_{n}}{\partial x^{2}}+\kappa\left(\sum_{k=1}^{N}\left|\psi_{k}\right|^{2}\right) \psi_{n}=0, \quad n=1, \ldots, N$

If the vector elements $\psi_{n}$ are further required to be orthogonal, they may be taken as modal functions in the selfconsistent multimode theory [9], and Eq. (3) gives the evolution of the modal functions. 
In Ref. [21], we show that Eq. (2) and its Hermitian conjugate can be derived as an operator condition $\left[\Delta^{(1)}, \Delta^{(2)}\right]=0$ by using the dressing operators, cf. [22],

$\Delta^{(1)}=\tilde{I}\left(i \frac{\partial}{\partial z}+p \frac{\partial^{2}}{\partial x^{2}}\right)+2 p\left[\begin{array}{cc}|\psi\rangle\langle\psi| / l_{1} & \left|\psi_{x}\right\rangle \\ \left\langle\psi_{x}\right| & -\langle\psi \mid \psi\rangle / l_{2}\end{array}\right]$

and

$$
\Delta^{(2)}=\tilde{I}\left[\begin{array}{ll}
l_{1} & 0 \\
0 & l_{2}
\end{array}\right] \frac{\partial}{\partial x}+\left[\begin{array}{cc}
0 & -|\psi\rangle \\
\langle\psi| & 0
\end{array}\right],
$$

where

$$
\tilde{I}=\left[\begin{array}{cc}
|1\rangle\langle 1| & 0 \\
0 & 1
\end{array}\right]
$$

is a generalization of the unit matrix with the identity ket $|1\rangle$. These operators depend on a single parameter $p$ such that, after rescaling $z \rightarrow z / \beta$, we have $\beta \kappa=$ $2 /\left(l_{1} l_{2}\right)=8 p^{2} /\left(1-p^{2}\right)$, with $l_{1}=(1-p) / 2 p \quad$ and $l_{2}=(1+p) / 2 p$.

If the operator pair given by Eqs. (4) and (5) is written as $\Delta^{(1)}=\tilde{I} i \frac{\partial}{\partial z}-\hat{A}$ and $\Delta^{(2)}=\hat{L}$, we recover the familiar Lax representation [3] with the two equations

$$
\begin{gathered}
\hat{L} \phi=\lambda \phi, \\
i \frac{\partial \phi}{\partial z}=[\hat{A}+g(\hat{L})] \phi,
\end{gathered}
$$

where $\lambda$ is the spectral eigenvalue, $\phi$ is the corresponding eigenfunction, and $g(\hat{L})$ is an arbitrary function of the operator $\hat{L}$; cf. [23]. The operator condition $\left[\Delta^{(1)}, \Delta^{(2)}\right]=$ 0 is then replaced by the equation

$$
i \frac{\partial \hat{L}}{\partial z}+[\hat{L}, \hat{A}]=0,
$$

and the spectrum is constant due to $\hat{A}$ being Hermitian. The integrability of the nonlinear evolution equation (2) follows from its representation as a Lax pair.

The eigenvalue problem for the scattering operator Eq. (7), viz.,

$$
\hat{L}\left[\begin{array}{l}
\phi_{1} \\
\phi_{2}
\end{array}\right]=\lambda\left[\begin{array}{l}
\phi_{1} \\
\phi_{2}
\end{array}\right],
$$

can, using the substitutions $\zeta=i \lambda /\left(2 l_{1} l_{2}\right)$ and

$$
\begin{aligned}
& \phi_{1}=\sqrt{l_{2}} \exp \left[-i\left(l_{1}+l_{2}\right) \zeta x\right] v_{1}, \\
& \phi_{2}=\sqrt{l_{1}} \exp \left[-i\left(l_{1}+l_{2}\right) \zeta x\right] v_{2},
\end{aligned}
$$

be written as the Zakharov-Shabat problem [2]

$$
\begin{aligned}
& \frac{\partial v_{1}}{\partial x}=-i \zeta v_{1}+\frac{1}{\sqrt{l_{1} l_{2}}}|\psi\rangle v_{2}, \\
& \frac{\partial v_{2}}{\partial x}=-\frac{1}{\sqrt{l_{1} l_{2}}}\langle\psi| v_{1}+i \zeta v_{2} .
\end{aligned}
$$

Normalizing $|\psi\rangle / \sqrt{l_{1} l_{2}} \rightarrow|\psi\rangle$, we seek a solution $v_{2}=$ $\exp \left[i \zeta x-i \omega(\zeta) z-i \zeta \int_{-\infty}^{x} \nu(t, z) d t\right]$, where $\omega(\zeta)$ satisfies the dispersion relation $\omega=4 p l_{1}^{2} \zeta^{2}$. According to Eq. (8), this implies that

$$
\frac{\partial}{\partial z} \int_{-\infty}^{\infty} \nu(x, z) d x=0
$$

since $|\psi\rangle \rightarrow 0$ as $x \rightarrow \infty$ and a Riccati equation for $\nu$ is obtained from Eq. (12), viz.,

$$
i \zeta \frac{\partial \nu}{\partial x}=\langle\psi \mid \psi\rangle+i \zeta\left\langle\psi_{x}\right|\left\langle\left.\psi\right|^{-1} \nu+2 \zeta^{2} \nu-\zeta^{2} \nu^{2},\right.
$$

where $\left\langle\left.\psi\right|^{-1}\right.$ is an inverse operator such that $\langle\psi|\left\langle\left.\psi\right|^{-1}=\right.$ $\left\langle\left.\psi\right|^{-1}\langle\psi|=1\right.$.

The solution of Eq. (14) is a generating function for an infinite set of conserved quantities $\int_{-\infty}^{\infty} w_{n}(x) d x$, with $w_{n}$ defined by $\nu=-\frac{1}{2 \zeta^{2}} \sum_{n=0}^{\infty}\left(\frac{i}{2 \zeta}\right)^{n} w_{n}$ and satisfying the recursion relation

$$
w_{n+1}=\frac{\partial w_{n}}{\partial x}-\left\langle\psi_{x}\right|\left\langle\left.\psi\right|^{-1} w_{n}+\sum_{k=0}^{n-1} w_{k} w_{n-1-k}\right.
$$

with $w_{0}=\langle\psi \mid \psi\rangle$.

The first six expressions obtained from Eq. (15) are given by

$$
\begin{gathered}
w_{0}=\langle\psi \mid \psi\rangle, \\
w_{1}=\left\langle\psi \mid \psi_{x}\right\rangle, \\
w_{2}=\left\langle\psi \mid \psi_{x x}\right\rangle+(\langle\psi \mid \psi\rangle)^{2} \\
w_{3}=\left\langle\psi \mid \psi_{x x x}\right\rangle+\langle\psi \mid \psi\rangle\left\langle\psi_{x} \mid \psi\right\rangle+4\langle\psi \mid \psi\rangle\left\langle\psi \mid \psi_{x}\right\rangle \\
w_{4}=\langle\psi \\
+\left\langle\psi \mid \psi_{x x x x}\right\rangle+3\left\langle\psi_{x} \mid \psi\right\rangle\left\langle\psi \mid \psi_{x}\right\rangle+3\langle\psi \mid \psi\rangle\left\langle\psi_{x} \mid \psi_{x}\right\rangle \\
+5\left(\langle\psi \mid \psi\rangle \psi_{x x}|\psi\rangle+6\langle\psi \mid \psi\rangle\left\langle\psi \mid \psi_{x x}\right\rangle\right. \\
w_{5}=2(\langle\psi \mid \psi\rangle)^{3}, \\
+\left\langle\psi \mid \psi \psi_{x x x x}\right\rangle+4\left\langle\psi \mid \psi \psi_{x}\right\rangle\left\langle\psi \psi_{x x} \mid \psi\right\rangle+11\left\langle\psi \mid \psi_{x}\right\rangle\left\langle\psi_{x} \mid \psi_{x}\right\rangle \\
+6\left\langle\psi \psi_{x} \mid \psi\right\rangle\left\langle\psi \mid \psi_{x x}\right\rangle+6\langle\psi \mid \psi\rangle\left\langle\psi_{x} \mid \psi_{x x}\right\rangle \\
+4\langle\psi \mid \psi\rangle\left\langle\psi_{x x} \mid \psi_{x}\right\rangle+\langle\psi \mid \psi\rangle\left\langle\psi_{x x x} \mid \psi\right\rangle \\
+8\langle\psi \mid \psi\rangle\left\langle\psi \mid \psi_{x x x}\right\rangle+18\left\langle\psi \mid \psi \psi_{x}\right\rangle\left\langle\psi \mid \psi_{x x}\right\rangle \\
+6(\langle\psi \mid \psi\rangle)^{2}\left\langle\psi_{x} \mid \psi\right\rangle+16(\langle\psi \mid \psi\rangle)^{2}\left\langle\psi \mid \psi_{x}\right\rangle,
\end{gathered}
$$

with the first three invariants having the physical interpretation of energy, momentum, and Hamiltonian conservation. Note that the operator $\left\langle\left.\psi\right|^{-1}\right.$ acts only on $\langle\psi|$ and should be distributed symmetrically, e.g., $\left\langle\psi_{x}\right|\left\langle\left.\psi\right|^{-1} \times\right.$ $\left[\langle\psi \mid \psi\rangle\left\langle\psi \mid \psi_{x}\right\rangle\right]=\frac{1}{2}\left\langle\psi_{x} \mid \psi\right\rangle\left\langle\psi \mid \psi_{x}\right\rangle+\frac{1}{2}\langle\psi \mid \psi\rangle\left\langle\psi_{x} \mid \psi_{x}\right\rangle$.

The above invariants can be directly calculated in terms of the coherent density function or the modal functions of the self-consistent multimode theory for infinite- and finite-dimensional bra-ket vectors, respectively. 
We will now establish how to express the invariants in terms of the mutual coherence and Wigner distribution functions. The latter are often more convenient to use, since they provide direct information about the overall degree of coherence of the field. In our bra-ket notation, the mutual coherence function is given by

$$
K\left(x_{1}, x_{2}, z\right)=\left\langle\psi\left(x_{1}, z\right) \mid \psi\left(x_{2}, z\right)\right\rangle
$$

and satisfies the evolution equation [10]

$$
i \frac{\partial K}{\partial z}+\beta\left(\frac{\partial^{2} K}{\partial x_{2}^{2}}-\frac{\partial^{2} K}{\partial x_{1}^{2}}\right)+\kappa\left[I\left(x_{2}, z\right)-I\left(x_{1}, z\right)\right] K=0
$$

with the intensity $I(x, z)=K(x, x, z)$.

The Wigner distribution function is obtained from the mutual coherence function, by transforming to coordinates $x=\left(x_{1}+x_{2}\right) / 2$ and $\xi=x_{1}-x_{2}$ and then taking the Fourier transform with respect to $\xi$, viz.,

$$
\rho(x, p, z)=\frac{1}{2 \pi} \int_{-\infty}^{\infty}\langle\psi(x+\xi / 2) \mid \psi(x-\xi / 2)\rangle \exp (i p \xi) d \xi,
$$

and satisfies the Wigner-Moyal equation [11]

$$
\frac{\partial \rho}{\partial z}+2 \beta p \frac{\partial \rho}{\partial x}+2 \kappa\langle\psi \mid \psi\rangle \sin \left(\frac{1}{2} \frac{\leftarrow}{\partial x} \frac{\vec{\partial}}{\partial p}\right) \rho=0,
$$

where $\langle\psi \mid \psi\rangle=\int_{-\infty}^{\infty} \rho d p$ and the sine operator is defined by its series expansion, with the arrows indicating the direction of application of the derivatives.

From the definition (22), it follows that the conservation laws may be expressed in the mutual coherence formalism using the relation

$$
\left.\frac{\partial^{m+n}}{\partial x_{1}^{m} \partial x_{2}^{n}} K\left(x_{1}, x_{2}, z\right)\right|_{x_{1}=x_{2}=x}=\left\langle\frac{\partial^{m} \psi}{\partial x^{m}} \mid \frac{\partial^{n} \psi}{\partial x^{n}}\right\rangle .
$$

In the Wigner formalism, we instead make use of the relation

$$
\int_{-\infty}^{\infty}\left(\frac{1}{2} \frac{\partial}{\partial x}-i p\right)^{m}\left(\frac{1}{2} \frac{\partial}{\partial x}+i p\right)^{n} \rho d p=\left\langle\frac{\partial^{m} \psi}{\partial x^{m}} \mid \frac{\partial^{n} \psi}{\partial x^{n}}\right\rangle
$$

which follows from

$$
\begin{aligned}
& \int_{-\infty}^{\infty}\left(\frac{1}{2} \frac{\partial}{\partial x}-i p\right)^{m}\left(\frac{1}{2} \frac{\partial}{\partial x}+i p\right)^{n} \rho d p \\
& \quad=\left.\int_{-\infty}^{\infty}\left(\frac{1}{2} \frac{\partial}{\partial x}+\frac{\partial}{\partial \xi}\right)^{m}\left(\frac{1}{2} \frac{\partial}{\partial x}-\frac{\partial}{\partial \xi}\right)^{n} \rho e^{-i p \xi} d p\right|_{\xi=0} \\
& =\left.\frac{\partial^{m+n}}{\partial x_{1}^{m} \partial x_{2}^{n}} \int_{-\infty}^{\infty} \rho e^{-i p \xi} d p\right|_{\xi=0} \\
& =\left.\frac{\partial^{m+n}}{\partial x_{1}^{m} \partial x_{2}^{n}} K\left(x_{1}, x_{2}, z\right)\right|_{x_{1}=x_{2}=x}
\end{aligned}
$$

by a change of variables and by applying the inverse Fourier transform concomitant to Eq. (24). For instance, using Eq. (18), the Hamiltonian invariant for the WignerMoyal equation (25), $\int_{-\infty}^{\infty} w_{2} d x$, becomes

$$
\int_{-\infty}^{\infty}\left[\int_{-\infty}^{\infty} p^{2} \rho d p-\left(\int_{-\infty}^{\infty} \rho d p\right)^{2}\right] d x=\text { const. }
$$

The invariance of this integral may, remembering the normalization $\beta \kappa\langle\psi \mid \psi\rangle \rightarrow 2\langle\psi \mid \psi\rangle$, be verified by taking the appropriate moments of Eq. (25).

In conclusion, we have shown that the nonlinear evolution equations governing the propagation of partially coherent waves in noninstantaneous Kerr media are integrable and have an infinite number of conserved quantities. A recursive relation to generate these conservation laws has been presented, as well as relations allowing them to be expressed in either formalism.

[1] M.J. Ablowitz and H. Segur, Solitons and Inverse Scattering Transform, SIAM Studies in Applied Mathematics Vol. 4 (SIAM, Philadelphia, PA, 1981).

[2] V. E. Zakharov and A. B. Shabat, Sov. Phys. JETP 34, 62 (1972).

[3] P. D. Lax, Commun. Pure Appl. Math. 21, 467 (1968).

[4] M. Mitchell, Z. Chen, M. Shih, and M. Segev, Phys. Rev. Lett. 77, 490 (1996).

[5] D. N. Christodoulides, T. H. Coskun, M. Mitchell, and M. Segev, Phys. Rev. Lett. 78, 646 (1997).

[6] D. N. Christodoulides, E. D. Eugenieva, T. H. Coskun, M. Segev, and M. Mitchell, Phys. Rev. E 63, 035601 (2001).

[7] M. Lisak, L. Helczynski, and D. Anderson, Opt. Commun. 220, 321 (2003).

[8] Y.S. Kivshar and G. P. Agraval, Optical Solitons: From Fibers to Photonic Crystals (Academic, San Diego, 2003).

[9] M. Mitchell, M. Segev, T.H. Coskun, and D. N. Christodoulides, Phys. Rev. Lett. 79, 4990 (1997).

[10] G. A. Pasmanik, Sov. Phys. JETP 39, 234 (1974).

[11] B. Hall, M. Lisak, D. Anderson, R. Fedele, and V.E. Semenov, Phys. Rev. E 65, 035602 (2002).

[12] V. G. Makhankov and O. K. Pashaev, Theor. Math. Phys. 53, 979 (1982).

[13] N. Akhmediev, W. Krolikowski, and A. W. Snyder, Phys. Rev. Lett. 81, 4632 (1998).

[14] N. Akhmediev and A. Ankiewicz, Chaos 10, 600 (2000).

[15] A. C. Scott, Phys. Scr. 29, 279 (1984).

[16] E. P. Bashkin and A. V. Vagov, Phys. Rev. B 56, 6207 (1997).

[17] R. Fedele, P. K. Shukla, M. Onorato, D. Anderson, and M. Lisak, Phys. Lett. A 303, 61 (2002).

[18] W. Craig, D. M. Henderson, M. Oscamou, and H. Segur, Math. Comput. Simul. 74, 135 (2007).

[19] M. Onorato, A. R. Osborne, and M. Serio, Phys. Rev. Lett. 96, 014503 (2006).

[20] P. K. Shukla, I. Kourakis, B. Eliasson, M. Marklund, and L. Stenflo, Phys. Rev. Lett. 97, 094501 (2006).

[21] T. Hansson, D. Anderson, and M. Lisak, Phys. Rev. E 84, 056601 (2011).

[22] V.E. Zakharov and A. B. Shabat, Funct. Anal. Appl. 8, 3 (1974).

[23] S. V. Manakov, Zh. Eksp. Teor. Fiz. 65, 505 (1973). 